\title{
Cardiac surgery during the times of COVID-19
}

\author{
Kirun Gopal ${ }^{1}$ (D) Praveen Kerala Varma ${ }^{1}$ (iD \\ Received: 2 May 2020 / Revised: 29 June 2020 / Accepted: 30 June 2020 / Published online: 20 July 2020 \\ (C) Indian Association of Cardiovascular-Thoracic Surgeons 2020
}

\section{Dear Editor,}

The unexpected occurrence of the COVID-19 pandemic has thrown normal life out of gear and there seems to be no immediate end in sight. Normal life has changed and the role of cardiac surgery during this time is still evolving. Potential dearth of hospital beds, possible unnecessary exposure of hospital staff to infection, and over utilization of scarce personal protection equipment (PPE) resources are issues of concern. Also we really do not know yet what is the added risk of a COVID-19 infection to a cardiac surgical patient.

Most centers had stopped or restricted their elective cases as advised by the Ministry of Health and Family Welfare [1]. The rationale to defer truly elective cardiac surgical procedures includes exposing elective patients to the virus in the hospital, exposure of hospital staff to an asymptomatic COVID + elective patient, utilization of intensive care unit (ICU) beds and ventilators for elective surgery, and potential wastage of PPE doing elective procedures.

However, continuing to defer all elective surgeries, we may reach a situation where a lot of patients may die of their original condition. So, departments have tentatively restarted their work. With increasing COVID cases, questions persist, and in such tumultuous circumstances, it is better if we can formulate a broad guideline for us. Factors to help in decision-making can be discussed under 3 headings.

\section{Patient factors}

In these circumstances, following normal guidelines to operate may not be possible and some sort of clinical judgment will be needed to stratify patients in order to ensure rapid

Kirun Gopal

kirungopal@gmail.com

1 Department of Cardiovascular and Thoracic Surgery, Amrita Institute of Medical Sciences, Amrita Vishwa Vidyapeetham (Amrita University), Kochi, Kerala, India turnover of ICU beds and ventilators and prevent chronic patients being stuck in the ICU.

\section{Institution factors}

The location, referral pattern, and standing in the community may all influence the future COVID burden of a particular institution. The availability of ICU beds, ventilators, pulmonologists, and anesthetists varies between hospitals.

\section{COVID factors}

The COVID pandemic is still evolving in India. We are not yet officially in the community transmission phase and exponential growth has still not occurred. But if exponential growth does happen, this will be the most important factor that determines the protocol for operating on cardiac surgical patients. In this situation, separating institutional loads into tiers, as mentioned in the Society of Thoracic Surgeons (STS) guidelines, may serve as a guide to decide which cardiac procedures to do [2].

\section{General recommendations}

Pathways should be institution specific. We propose certain suggestions, which can be taken into consideration when formulating local protocols.

\section{Staff rotation}

Reduction in the volume of cases being done will allow for a skeletonization of staff at all levels and divide the group into treatment teams, in order to prevent the entire group being put out of service, if one gets positive. 


\section{Testing for COVID before surgery}

Mandatory testing for COVID in all patients coming for surgery is the ideal situation. But in the current scenario, testing kit availability is an issue. One will have to go by epidemiological factors to do testing. However, we still do not know what should be the ideal testing protocol as the nasopharyngeal polymerase chain reaction (PCR) swab can be negative due to a number of reasons, including improper technique of sampling, low viral load, or mutations in the viral genome [3]. The value of antibody tests is also being evaluated by the Indian Council of Medical Research (ICMR) [4]. Noncontrast computed tomography (CT) chest screening for ground glass opacities as a marker for COVID disease has also been used in some centers and the validity and feasibility of this test as a screening tool are also yet to be validated [5].

\section{Improving patient communication}

In these distressing times for both patients and doctors, open lines of communication should be established with patients in whom surgery is deferred in view of their elective status. Proper explanation of the current situation and the need to defer surgery should be explained including the risk to the patient. Embracing telemedicine in one's institution will facilitate this new mode of following up patients.

\section{Formulating an institution-specific pathway}

Stratification of patients should take into consideration COVID wave, institution preparedness, and patient risk. We feel that in the current scenario, one should only be doing really indicated procedures and that would broadly entail coronary artery bypass grafting $(\mathrm{CABG})$, where there is tight coronary disease with a significant area of myocardium at risk, symptomatic severe aortic stenosis, valve lesions in medically refractory heart failure and endocarditis in failure or high risk for embolism, and symptomatic aortic aneurysms with risk of rupture.

In the early phase I of the COVID pandemic, which we are currently in, we can probably go ahead with these abovementioned procedures, with some latitude in patients being operated on as the disease burden is low. However, it is probably advisable to use some form of PPE like the N95 mask. Phase 1 can be defined by a no or low COVID burden in the hospital.

Phase II is when the COVID burden in the hospital is on the upswing and ICU and ventilated patients are increasing daily. At this point, hospital transmission of the disease becomes more of a possibility and so logically, criteria to operate should be more strict and justifiable.

Phase III is when COVID burden is nearing or at full capacity of the hospital. At this stage, ICU beds and ventilators will be at a premium. At this stage, one should really be operating on patients who would die if surgery is not performed like acute type A aortic dissection, coronary artery disease (CAD) with rest angina despite maximal medication and not amenable to percutaneous coronary intervention (PCI), valve patients in medically refractory failure, and ruptured or impending rupture aortic aneurysms.

\section{Conclusion}

In conclusion, cardiac surgery during the time of COVID-19 is an evolving situation. Decisions to operate should be based on patient factors, institution capacity, and disease burden in the community.

Funding No funding was received to assist with the preparation of this manuscript.

\section{Compliance with ethical standards}

Conflict of interest The authors declare that they have no conflicts of interest.

Ethics approval As no human subjects were involved in the preparation of this manuscript, no ethics committee approval was obtained.

\section{References}

1. Ministry of Health and Family Welfare resource - Advisory for Hospitals and Medical Institutions - dated 20/3/2020. Accessed 28 Apr 2020.

2. Haft JW, Atluri P, Alawadi G, et al. Adult cardiac surgery during the COVID-19 pandemic: a tiered patient triage guidance statement. Ann Thorac Surg. 2020. https://doi.org/10.1016/j.athoracsur.2020. 04.003 .

3. Udugama B, Kadhiresan P, Kozlowski HN, et al. Diagnosing COVID-19: the disease and tools for detection. ACS Nano. 2020;14:3822-35.

4. Indian Council of Medical Research Resource - Revised Advisory to state of Rapid Antibody Test (dated 27/4/2020). Accessed 28 Apr 2020.

5. Dangis A, Gieraerts C, Bruecker YD, et al. Accuracy and reproducibility of low-dose submillisievert chest CT for the diagnosis of COVID-19. Radiol Cardiothorac Imaging. 2020;2:e200196.

Publisher's note Springer Nature remains neutral with regard to jurisdictional claims in published maps and institutional affiliations. 